\title{
Y a-t-il une app pour ça?
}

\author{
par Douglas Sellinger
}

$\mathrm{L}_{\mathrm{s}}^{\circ}$ orsqu'est née notre fille unique il y a près de 16 ans d'une grossesse à risque élevé, les deux premières choses que nous avons achetées étaient une minifourgonnette et un téléphone cellulaire muni d'une antenne externe. Peu après, nous avons pris la route de l'électronique avec un accès commuté à Internet.

Aujourd'hui, notre accès Internet haute vitesse sans fil relie nos ordinateurs portatifs et de bureau, iPod et téléphones intelligents au reste du monde. Ma fille est complètement perdue si elle ne peut texter, mettre à jour sa page Facebook et chatter avec ses amis et camarades de classe tout en écoutant de la musique et en jouant à des jeux. La technologie est devenue omniprésente dans notre maison et fait partie intégrante de la vie de ma fille. Et comme si la technologie que nous avons à la maison n'était pas suffisante, j'ai passé une grande partie des 15 dernières années dans des équipes qui ont fait entrer la technologie dans le service de pharmacie et le réseau de santé où je travaille.

La sécurité des patients et l'utilisation sécuritaire des médicaments sont d'importants moteurs de l'avancée de la technologie dans les pharmacies d'hôpitaux. La codification à barres pour l'administration des médicaments au chevet des patients, la saisie électronique des ordonnances par les médecins, les dossiers médicaux électroniques, les cabinets automatisés décentralisés et les logiciels d'aide à la décision clinique lient de plus en plus les pharmaciens et les pharmacies à une panoplie de postes de travail, tablettes, miniportatifs et téléphones intelligents. Vous pouvez utiliser une app (application) avec votre base de données préférée d'information sur les médicaments, qui vous permet d'utiliser votre téléphone intelligent pour la consulter, même si vous n'avez pas accès à un poste de travail ou à un portatif.

Mais ces avancées en technologie ne sont pas sans faille et n'ont pas rendu pour autant les pharmaciens « redondants ». Par exemple, Saverno et ses collaborateurs ( $\mathrm{Am}$ Med Inform Assoc 2011;18[1]:32-37) ont traité de ce que tout pharmacien éprouvant une lassitude d'alerte savait déjà : les logiciels actuels d'aide à la prise de décision clinique alertent les utilisateurs sur trop d'interactions médicament-médicament sans pertinence et parfois oublient de signaler des interactions qui leur seraient pourtant essentielles. Les logiciels d'aide à la décision clinique s'améliorent, mais beaucoup reste à faire avant qu'ils ne soient en mesure de procurer aux pharmaciens et aux prescripteurs des alertes sur les médicaments "intelligentes " adaptées à chaque client.

À l'instar du pharmacien hospitalier traduisant les alertes d'aide à la décision en information utile, les trousses à outils "Hit the Target " développées par la Société canadienne des pharmaciens d'hôpitaux (SCPH) dans le cadre du projet SCPH 2015 réduiront la surinformation. La première trousse, «From Paper to Practice: Incorporating Evidence into Your Pharmacy Practice ", est maintenant disponible en ligne, avec deux autres ("Complex Inpatients Need Medication Experts " et "One Dose at a Time») qui le seront sous peu. Ces trousses proposent un modèle de mise en ouvre, de mesures d'évaluation et de références pour aider à mettre de l'avant les changements requis pour atteindre leurs objectifs respectifs du projet SCPH 2015.

Outre ces trousses d'outils SCPH 2015, j'étais emballé de voir l'ajout du nouveau portail extranet MY.CSHP.ca! Je suis impatient de voir l'évolution de la présence de la SCPH en ligne afin de toujours mieux répondre aux besoins de communication et d'information de ses membres. Dans cette ère riche en technologies, améliorer les façons dont les membres peuvent «se connecter " à la SCPH vient bonifier l'utilité de la Société et les avantages pour les membres, et permettre le partage tout à fait singulier d'idées porteuses d'améliorations qui, je crois, distinguent les pharmaciens du Canada. Alors, cette " app $\mathrm{SCPH}$ " pour votre iPhone, votre Android ou votre BlackBerry est-elle si loin?

[Traduction par l'éditeur]

Douglas Sellinger, BSP, MALT, assumera le rôle de président désigné de la Société canadienne des pharmaciens d'hôpitaux (SCPH) et se chargera de la liaison externe après l'Assemblée générale annuelle de la SCPH en août 2011. 